\title{
Experimental Measurements of Capacity for MIMO Indoor Wireless Channels
}

\author{
A. Lee Swindlehurst, Gus German, Jon Wallace, \\ Michael Jensen ${ }^{1}$ \\ Dept. of Electrical \& Computer Engineering \\ Brigham Young University \\ 459 Clyde Building \\ Provo, UT 84602 \\ e-mail: swindle@ee.byu.edu
}

\begin{abstract}
This paper presents the results of experiments obtained with a narrowband, multiple-input multiple-output (MIMO) channel probe in an indoor of fice/laboratory environment. The system employs ten transmit and ten receive antennas, and computes the $10 \times 10$ narrowband channel matrix at $2.4 \mathrm{GHz}$ every 80 milliseconds. We use the data to study the distribution of the channel coefficients, their temporal variability, and the resulting effects on channel capacity. We also compare the empirical results with those predicted by WiSE, a state-of-the-art channel simulator based on ray-tracing from Lucent Technologies.
\end{abstract}

\section{INTRODUCTION}

The promise of achieving significant increases in wireless channe capacity using MIMO architectures [1, 2] has recently sparked considerable interest. Numerous space-time coding algorithms have been developed in order to realize the potential throughput gains. For example, Lucent Technologies' BLAST system has been demonstrated to attain throughputs of $20-40 \mathrm{bits} / \mathrm{sec} / \mathrm{Hz}$ in controlled laboratory experiments $[3,4]$. Using a $4 \times 4$ experimental system in an outdoor mobile environment, AT\&T Labs has shown that the theoretical limit of a fourfold increase in capacity is nearly. always achieved [5]. Other experiments have also shown promising results $[6,7 ; 8]$.

In this paper, we describe experimental results obtained with a $10 \times 10$ element narrowband channel probing system implemented at $2.4 \mathrm{GHz}$ in an indoor office/laboratory environment. The system transmits and receives all data in parallel (ie, no sequential data transmission or sampling is used), and takes a snapshot of the $10 \times 10$ channel matrix every $80 \mathrm{~ms}$. We use data collected in several different rooms with various array orientations to empirically determine the statistics of the channel coefficients and the channel capacity. Of particular importance is the effect of channel time variations on capacity. We also compare the empirically measured capacity with that predicted by a state-of-the-art channel simulator (WiSE) provided by Lucent Technologies [9].

\section{Mimo Channel Measurement System •}

The experimental platform is a custom MIMO communications system with 10 transmit and 10 receive monopole antennas that can operate at frequencies between 0.8 and $6 \mathrm{GHz}$. Data for the experiments described in this paper were taken at $2.4 \mathrm{GHz}$. The system broadcasts 10 uniquely-coded and co-channel binary

${ }^{1}$ This work was supported by the National Science Foundation under Wireless Initiative Grant CCR 99-79452. phase shift keyed (BPSK) signals, one from each of the transnit antennas. The transmitter hardware consists of a custom raclio frequency (RF) system coupled with a digital pattern generator (DPG) and tunable microwave source. The RF chassis contains a backplane that distributes the local oscillator (LO) signal from the source, the binary sequences from the DPG, and DC povrer to up to 16 individual transmit cards. The LO signal is chenneled through a broadband resistive power divider to 16 surfece mount SMA connectors. The baseband signals and power are supplied via an edge-card connector. Each individual transrait card amplifies the LO signal and multiplies it with the supplied binary sequence to produce BPSK modulation. The resulting signal is amplified to $0.5 \mathrm{~W}$ and fed to one of the 10 transrait antennas.

The receiver downconverts the signal from each of the 10 receive antennas and stores the resulting sequences on a PC for post-processing. The receiver hardware consists of an RF chassis, a microwave source, and a PC for data acquisition. The RF chassis contains a backplane similar to that in the transinit system. Each receive card provides amplification and downconversion for the signal from one of the 10 receive antennas. The IF signals from all receive cards are low-pass filtered, amplified, and sampled on a 16-channel 1.25 Msample/s A/D card for storage on the PC. A block diagram of the complete MIMO system is depicted in Figure 1.

\section{Data Processing}

The raw data collected using the measurement platform is processed to obtain estimates of the time-variant channel matrix. The data processing consists of 3 steps: (1) code search, (2) carrier recovery, and (3) channel estimation.

1. Code Search - The first step in the data post-processing is to determine the alignment of the modulating codes The method begins by correlating the signal from one of the 10 receive antennas with a baseband representation of one of the transmit codes. An FF' of this result produces a peak at the IF when the known code and the code in the receive signal are aligned. If the signal carrying the specified code is weak, the maximum correlation may not occur at codeword alignment. To overcome this, our procedure searches over every combination of receive charine and codeword to ensure accurate code synchronization:

2. Carrier Recovery - A rough estimate of the intermediate frequency is obtained from the peak of the FFT taken during the code search process. This frequency is then refined using a subplex optimization loop that maximizes the magnitude of the Discrete Time Fourier Transform 


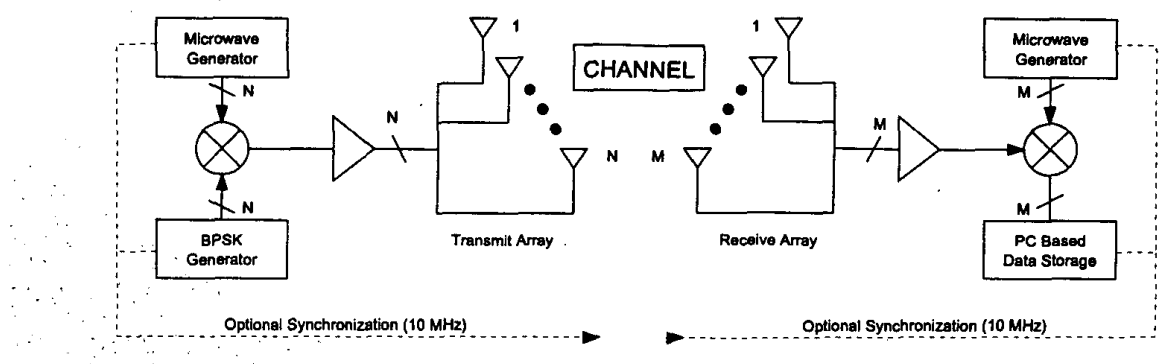

Figure 1: High level system diagram of the narrowband wireless MIMO measurement system.

(DTFT) of the despread signal (known aligned code multiplied by the receive signal). Following estimation of the frequency, the time evolution of the phase is recovered as follows: a recovery window is shifted along the despread receive signal at one sample increments. At each position, the signal is correlated against a complex sinusoid at the estimated IF frequency. The estimated phase at the center of the window is the angle of the complex result of the correlation. This phase estimate is finally smoothed by an averaging window.

3. Channel Estimation - The channel estimate is obtained by correlating the time- and frequency-aligned data from each receive antenna with the transmitted codes.

\section{WiSe Simulation Package}

Lucent Technologies has developed a 3-D predictive simulator for wireless channel propagation referred to as WiSE for Wireless System Engineering. It makes use of computational geometry, building environment descriptions, and a complex propagation model to predict the highest strength propagation path from arbitrary transmitter and receiver locations within a static environment [9]. Three dimensional ray-tracing is used to include the effects of frequency, polarization, the dielectric properties of encountered materials, diffraction around corners, and antenna directivity. To speed up the computationally intensive task of predicting all possible illumination paths from transmitter to receiver, only rays with power above a fixed threshold are perpetuated through the model. Also, rays which are outside of the three dimensional cone illuminated by reflections off of a particular wall interface are discarded. This simulation tool allows deterministic prediction of the impulse response of wireless channels for both time and angle of arrival. In addition, it permits insight into the physical structure of scatterers in the environment that contribute to arrival clustering by fully describing the path traveled by each individual ray from transmitter to receiver

We used the WiSE Simulation Package to simulate wireless channels at $2.4 \mathrm{GHz}$ in various portions of the W. W. Clyde Engineering Building located on the Brigham Young University Campus in Provo, Utah. The Clyde Engineering building is representative of steel-reinforced, cement block construction. The building geometry database needed for the simulations was taken from original architectural CAD files. To reduce the complexity of the geometry, it was assumed that the dominant paths in the channel impulse response remained in the same horizontal plane as the transmitter and receiver. Initially, this building model of a single floor consisted of over 2500 wall entities with

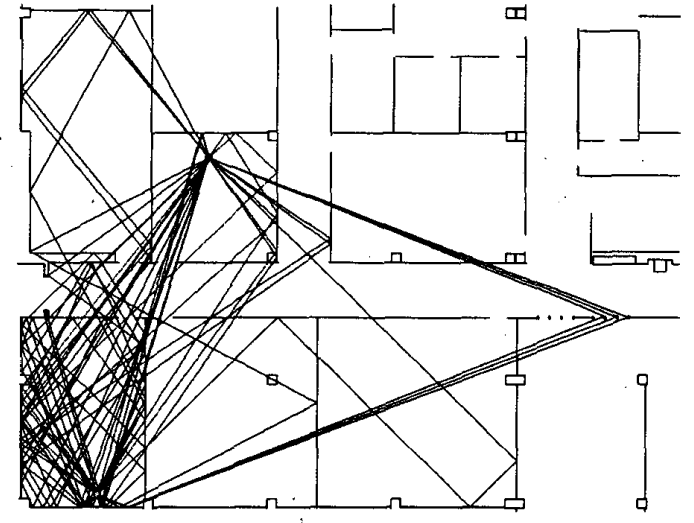

Figure 2: Typical Results of Ray Tracing Simulation for a single transmitter/receiver location pair.

uniform dielectric composition. Using this as a starting point, we condensed the model to focus on the location and properties of scatterers in the building. This resulted in a final model with much lower wall entity density and correspondingly lower simulation time with improved statistical results. An example of the ray-tracing results from these simulations is shown in Figure 2.

\section{ExPERIMENTAL Results}

Data were collected with the transmit and receive antenna arrays at 13 different pairs of locations in the Clyde Building (CB). All CB walls run north-south or east-west, and the axis of the arrays were always aligned in one of these two directions. Two experiments were conducted at each location, one with the arrays aligned, and one with arrays at right angles to each other. In each experiment, 4-5 ten second data sets were collected, with roughly 2 seconds between each set. The data in each set were used to estimate the $10 \times 10$ channel coefficient matrix every $80 \mathrm{~ms}$, which resulted in 124 channel estimates per set.

Figures 3-9 summarize some of the results obtained in processing the data. Figure 3 displays a histogram of the magnitude and phase of the elements of all channel matrices measured at all locations. Prior to forming the histogram, each $10 \times 10$ channel matrix was normalized so that the average modulus of its entries was unity. Also shown is an ideal Rayleigh density function with parameter $1 / 2$, which would correspond to the sum of two zero-mean independent Gaussian random variables (real and imaginary parts of the coefficient) with a variance of $1 / 2$ 


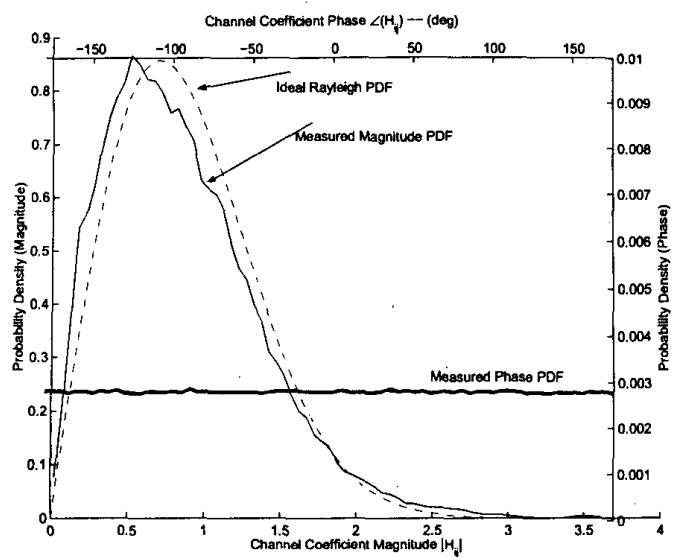

Figure 3: Empirical and Ideal Probability Density Functions for Channel Coefficient Magnitude and Phase.

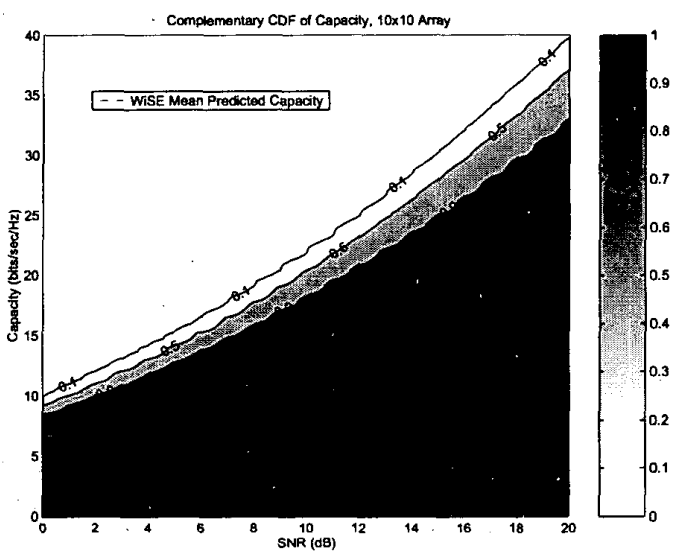

Figure 4: CDF of Capacity for $10 \times 10$ Array vs. SNR with Ray-Tracing Estimate.

each. The Rayleigh distribution provides a very accurate fit to the measured data, and the phase is essentially uniform.

Using the normalized empirical channel matrices, the capacity was calculated for each measurement at each location, and its complementary cumulative distribution function (CDF) is plotted in Figures 4 and 5. Figure 4 is for the full $10 \times 10$ MIMO channel, while Figure 5 shows the results for a $5 \times 5$ chànnel obtained by taking every other element from both arrays. As an example of how to interpret the plot, in Figure 4, the line labeled 0.9 is located at about $34 \mathrm{bits} / \mathrm{sec} / \mathrm{Hz}$ at 20 $\mathrm{dB}$ SNR. This means that the channel capacity at $20 \mathrm{~dB}$ SNR was measured to be above $34 \mathrm{bits} / \mathrm{sec} / \mathrm{Hz}$ in $90 \%$ of the trials involving the full 10-element arrays.

Superimposed on both plots is the average capacity predicted by the WiSE ray-tracing measurements at each SNR. Note that the measured capacity was on average greater than that predicted by WiSE in about $95 \%$ of the trials, which is not surprising since the WiSE simulation was not given information about the presence of additional scatterers such as desks, ta-

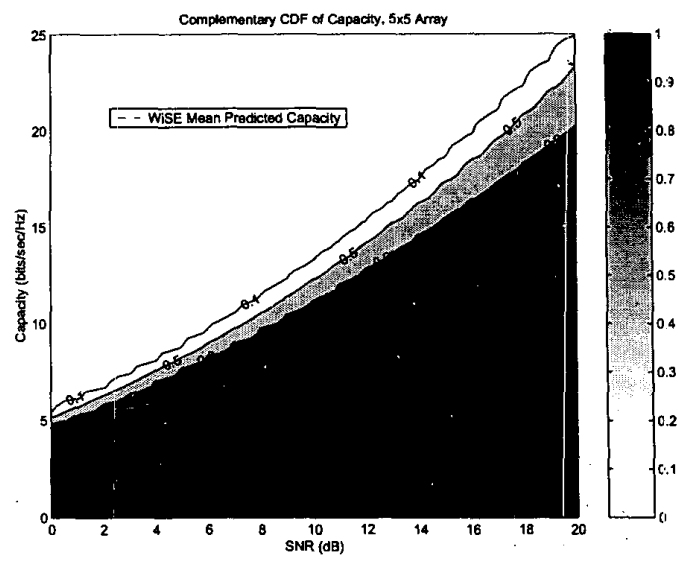

Figure 5: CDF of Capacity for $5 \times 5$ Array vs. SNR with Ray-Tracing Estimate.

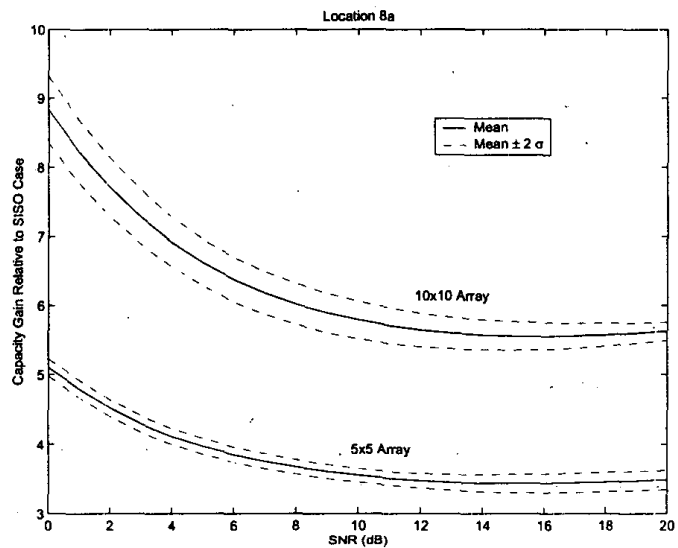

Figure 6: Relative Capacity Gain for $10 \times 10$ and $5 \times 5$ Arrays over SISO system vs. SNR, Location $8 \mathrm{a}$.

bles, computers, and people who were in the area at the time of the measurements. Nonetheless, the WiSE data does provide a fairly tight lower bound on the capacity that is expected. in a given location.

In Figure 6, we plot the relative gain in capacity obtained at one particular location (Location 8a) over that predicted for a single input, single output (SISO) system operating with the same total transmit power. For location $8 \mathrm{a}$, the transmit array was placed in room $498 \mathrm{CB}$ (dimensions $20 \mathrm{ft}$. by $30 \mathrm{ft}$.) and the receive array in $495 . \mathrm{CB}$ (dimensions $20 \mathrm{ft}$. by $20 \mathrm{ft}$.) both oriented in the same direction. However, the arrays were not pointing at one another since the rooms are located diagonally across an 8 foot wide hallway. The center of the rooms are separated by approximately 50 feet. No one was in the rooms when the measurements were taken, although people were present in adjacent rooms and walking down the hallway. Interestingly, the relative capacity gain decreases as the SNR increases.

Figures 7-9 give insight into the stationarity of the channel for Location $8 \mathrm{a}$. In Figure 7 we plot the relative capacity 


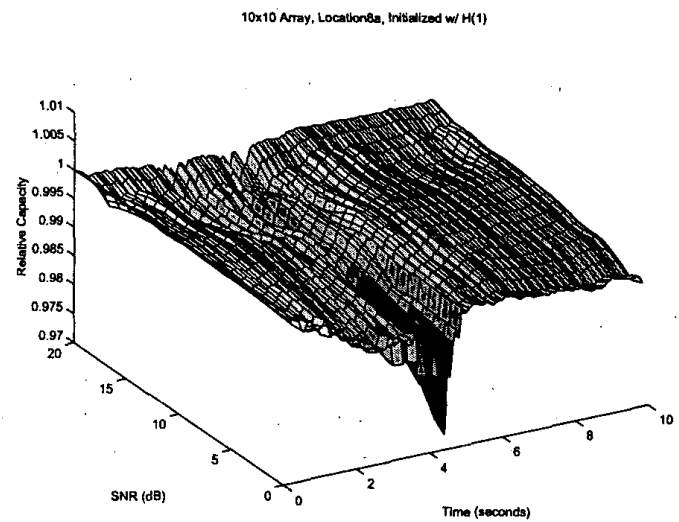

Figure 7: Relative Capacity with Time Using Water-Filling Solution for Initial Measured Channel Matrix, Location 8a.

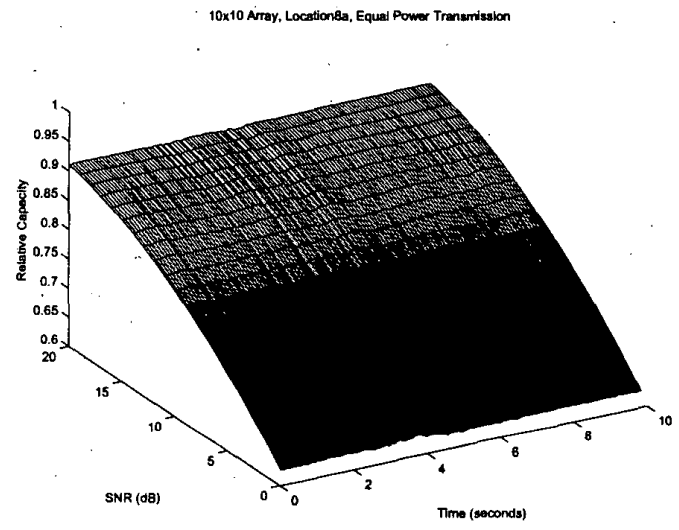

Figure 8: Relative Capacity with Time Using EquiPowered Transmission from All Antennas, Location 8a.

achieved by taking the first measured channel at time 0 , calculating the optimal transmit covariance matrix based on the water-filling solution, and then using this transmit covariance over the entire 10 second interval (the plot was generated by taking the average of the four 10-second data sets collected in this location). This results in, at worst, only an average $3 \%$ loss in capacity compared with using the optimal solution calculated at each time instant. Nearly all of the measurements were within $1 / 2 \%$ of the achievable capacity.

Figure 8 shows the loss in capacity over time if independent, equi-powered data streams are transmitted simultaneously from all antennas. The success of this scheme depends, of course, on the SNR; at high SNR, nearly optimal performance is achieved. At low SNR, there is a significant penalty. Again note that the achieved performance is essentially constant with time. Figure 9 shows the loss in capacity assuming that the water-filling solution is calculated based on the estimate of the channel matrix obtained with the WiSE simulation. A sizeable fraction of the capacity is still obtained even in this case, but not as much as if independent data are transmitted from each antenna.

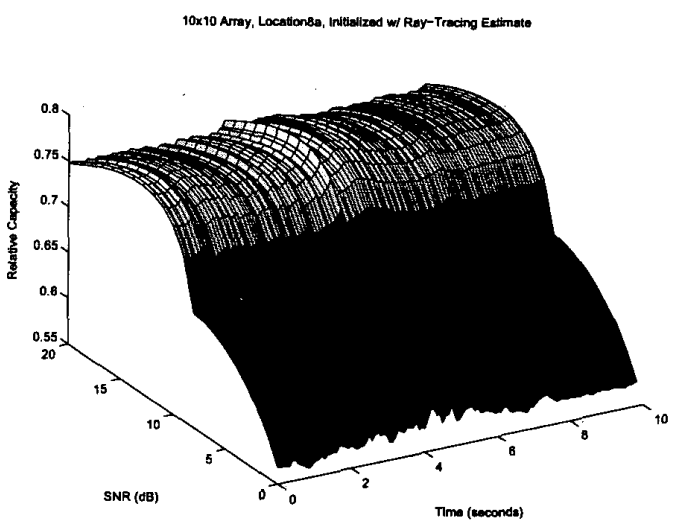

Figure 9: Relative Capacity with Time Using Water-Filling Solution for Ray-Tracing Estimate of Channel Matrix, Location 8a.

\section{References}

[1] I. Telatar, "Capacity of Multiple Antenna Gaussian Channels", ATET Technical Memorandum, June 1995.

[2] G. Foschini and M. Gans, "On Limits of Wireless Communications in a Fading Environment when Using Multiple Antennas", Wireless Personal Communicaitons, 6(3):311335, March 1998.

[3] P. Wolniansky, G. Foschini, G. Golden, and R. Valenzuela, "V-BLAST: An Architecture for Realizing Very High Data Rates Over the Rich Scattering Wireless Channel", In Proc. IEEE ISSSE Conf., Pisa, Italy, September 1998.

[4] G. Golden, G. Foschini, R. Valenzuela, and P. Wolniansky, "Detection Algorithm and Initial Laboratory Results using the V-BLAST Space-Time Communications Architecture", Electronics Letters, 35(1):14-15, Jan. 1999.

[5] C. Martin, J. Winters, and N. Sollenberger, "Multiple-Input Multiple-Output (MIMO) Radio Channel Measurements", In Proc. Vehic. Tech. Conf., Boston, MA, 2000.

[6] J. Wallace and M. Jensen, "A System for Measuring the Indoor and Outdoor MIMO Wireless Channel Response", In 2000 URSI/USNC Nat'l Radio Sci. Mtg. Digest, Salt Lake City, UT, July 2000.

[7] D. McNamara, M. Beach, P. Karlsson, and P. Fletcher, "Initial Characterization of Multiple-Input Multiple-Output (MIMO) Channels for Space-Time Communication", In Proc. Vehic. Tech. Conf., Boston, MA, 2000.

[8] J.-P. Kermoal, L. Schumacher, P. Mogensen, and K. Pedersen, "Experimental Investigation of Correlation Properties of MIMO Radio Channels for Indoor Picocell Scenarios", In Proc. Vehic. Tech. Conf., Boston, MA, 2000.

[9] S. Fortune, D. Gay, B. Kernighan, O. Landron, R. Valenzuela, and M. Wright, "WISE Design of Indoor Wireless Systems", IEEE Comp. Sci. and Eng., 2(1):58-68, Spring 1995. 